\title{
Economic benefits of subcutaneous rapid push versus intravenous immunoglobulin infusion therapy in adult patients with primary immune deficiency
}

\author{
A. Martin, ${ }^{1}$ L. Lavoie, ${ }^{2}$ M. Goetghebeur ${ }^{2} \&$ R. Schellenberg ${ }^{1,3}$ \\ ${ }^{1}$ St Paul's Hospital, Vancouver, BC, ${ }^{2}$ The LA-SER Group, Montreal, and ${ }^{3}$ Department of Medicine, James Hogg iCapture Centre for \\ Cardiovascular and Pulmonary Research, University of British Columbia, Vancouver, BC, Canada
}

Received 6 February 2012; accepted for publication 27 September 2012

\section{SUMMARY}

Objective: The objective of this study is to evaluate the economic benefits of immunoglobulin replacement therapy achieved subcutaneously (subcutaneous immunoglobulin, SCIG) by the rapid push method compared to intravenous infusion therapy (intravenous immunoglobulin, IVIG) in primary immune deficiency (PID) patients from the healthcare system perspective in the context of the adult SCIG home infusion program based at St Paul's Hospital, Vancouver, Canada.

Materials and methods: SCIG and IVIG options were compared in cost-minimisation and budget impact models (BIMs) over 3 years. Sensitivity analyses were performed for both models to evaluate the impact of varying modality of IVIG treatments and proportion of patients switching from IVIG to SCIG.

Results: The cost-minimisation model estimated that SCIG treatment reduced cost to the healthcare system per patient of $\$ 5736$ over 3 years, principally because of less use of hospital personnel. This figure varied between $\$ 5035$ and $\$ 8739$ depending on modality of IVIG therapy. Assuming 50\% of patients receiving IVIG switched to SCIG, the BIM estimated cost savings for the first 3 years at $\$ 1.308$ million or $37 \%$ of the personnel and supply budget. These figures varied between $\$ 1.148$ million and $\$ 2.454$ million (36 and $42 \%$ ) with varying modalities of IVIG therapy. If $75 \%$ of patients switched to SCIG, the reduced costs reached $\$ 1.962$ million or $56 \%$ of total budget.

Conclusion: This study demonstrated that from the health system perspective, rapid push home-based SCIG was less costly than hospital-based IVIG for immunoglobulin replacement therapy in adult PID patients in the Canadian context.

Correspondence: Dr Robert Schellenberg, Professor, Department of Medicine, James Hogg iCapture Centre for Cardiovascular and Pulmonary Research, University of British Columbia, St Paul's Hospital, 1081 Burrard St., Vancouver, BC, V6Z 1Y6 Canada. Tel.: +604 682 2344; fax: +604 8068351 ;

e-mail: bob.schellenberg@hli.ubc.ca
Key words: budget impact model, cost minimisation, IVIG, primary immune deficiencies, SCIG.

Primary immune deficiencies (PIDs) are a group of chronic disorders that can affect patients at various ages (Shehata et al., 2010). These disorders include agammaglobulinaemia, hyper-IgG syndrome, common variable immunodeficiency (CVID), transient hypogammaglobulinaemia of infancy and selective immunoglobulin deficiencies (Sorensen \& Moore, 2000). Prevalence of PID is estimated to be from one in two thousand to one in ten thousand of the general population in the United States (Turvey et al., 2009). Insufficient primary antibody production accounts for the majority of PID, which can result in serious opportunistic infections in affected patients (Sorensen \& Moore, 2000). Immunoglobulin replacement therapy has become the treatment of choice for PID patients for several decades (Berger, 2008). Immunoglobulin can be administered by intravenous or subcutaneous infusion. Intravenous immunoglobulin (IVIG) infusion is typically performed on a monthly basis in an outpatient setting (hospital), whereas subcutaneous immunoglobulin (SCIG) infusion can be self-administered one or more times a week by the patient at home (Berger, 2004; Lemieux et al., 2005). Similar efficacy in preventing infections has been reported between SCIG and IVIG with no difference in severity and length of infections (Chapel et al., 2000; Shehata et al., 2010). Although these two treatment options are associated with similar efficacy and safety profiles, (Chapel et al., 2000) switching from hospital-based IVIG to home-based SCIG was shown to significantly improve healthrelated quality of life (HRQoL) of adult PID patients (Gardulf et al., 2004; Kittner et al., 2006; Nicolay et al., 2006).

Among the SCIG administration options, a recent US study of a population of PID patients referred to an immunotherapy clinic reported that $71 \%$ of patients selected the rapid push method rather than pump infusion administration (Shapiro, 2010). The rapid push method was chosen less often by young children (2-10 years of age) but was the preferred method in teenagers and adults (Misbah et al., 2009; Shapiro, 2010). 
Healthcare resource utilisation differs markedly between SCIG and IVIG options. European economic studies performed in Sweden (Gardulf et al., 1995), Germany (Hogy et al., 2005), the UK (Liu et al., 2005) and France (Haddad et al., 2006; Beaute et al., 2010) reported that home-based SCIG was $25-75 \%$ less costly for the healthcare system than hospital-based IVIG. A Canadian study reported a cost difference of $<10 \%$ between the two options (Membe et al., 2008). In this study, immunoglobulin product formed $85 \%$ of the total cost of IVIG therapy and the same cost was applied to both IVIG and SCIG therapies (Membe et al., 2008). In studies from France and UK (Beaute et al., 2010; Liu et al., 2005), IVIG and SCIG costs were also equivalent but represented a smaller part, 70 and $58 \%$, respectively, of total costs of therapy. In studies from Germany and Sweden, the rationale to include immunoglobulin cost was supported by the lower cost of SCIG compared with IVIG in these countries (Hogy et al., 2005; Gardulf et al., 1995).

The objective of this study was to explore specifically the economic benefits of the rapid push method for SCIG compared with IVIG from the healthcare system perspective in the context of the adult SCIG home infusion program based at St Paul's Hospital, Vancouver, Canada.

\section{MATERIALS AND METHODS}

\section{Cost-minimisation analysis}

To compare SCIG and IVIG options in the context of the SCIG home infusion program based at St Paul's Hospital in Vancouver, a cost-minimisation analysis was performed, because current clinical knowledge indicates no difference in efficacy or side effect profiles between immunoglobulin therapy given subcutaneously and intravenously (Chapel et al., 2000). SCIG delivered as a rapid push rather than as conventional pump infusion was selected for the base case for this study because it is the most commonly used option in the SCIG home infusion program (winged needle butterfly infusion set). This method does not require an infusion pump and delivers SCIG by direct manual push from a syringe over short intervals determined by the patient's comfort level.

The analysis was performed from the perspective of the healthcare system and considered direct medical costs associated with both treatment options over a period of 3 years. The model compared SCIG and IVIG options for administration of immunoglobulin to adult PID patients. Treatment pathway for the base case is depicted in Fig. 1. The SCIG option requires three training sessions and includes four infusions a week using a winged needle butterfly infusion set (208 infusions per year). For IVIG, the base case represents average distribution of two current modalities in use at St Paul's Hospital, i.e. 13 (for two of three of the patients) or 17 visits (for one of three of the patients) annually and a 4 -h infusion session each visit.

Resource used for the base-case SCIG and IVIG options for the first 3 years of therapy are considered in the model shown in Table 1 and include supplies for rapid push SCIG and IVIG

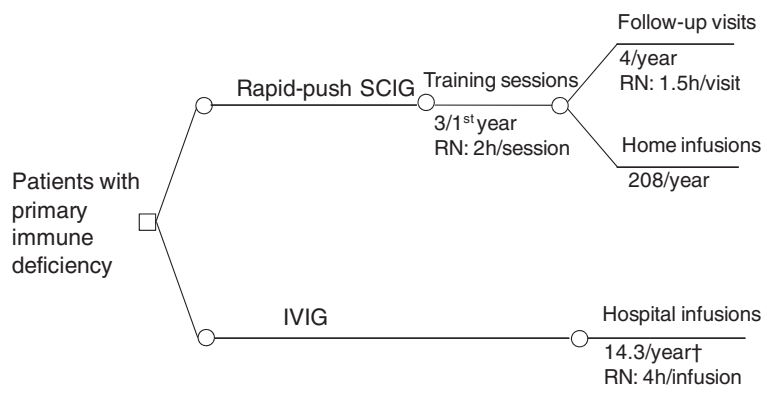

$\mathrm{RN}$ : registered nurse

† weighted average (2/3 patients: 13 visits, $1 / 3$ patients : 17 visits)

Fig. 1. Treatment pathway for the base case models comparing rapid push SCIG and IVIG treatment in primary immune deficiency (PID) based on current practice at the adult SCIG home infusion program, St Paul's Hospital, Vancouver.

Table 1. Resource use for base case models

\begin{tabular}{lcc}
\hline Resources & SCIG & IVIG \\
\hline First year & 208 & \\
$\quad$ Infusion supplies (day) & - & $14 \cdot 3$ \\
RN (h) & 12 & $57 \cdot 2$ \\
RN/manager (h) & - & - \\
Unit clerk (h) & - & $5 \cdot 72$ \\
Scheduling clerk (h) & - & $7 \cdot 15$ \\
Ward aid (h) & & $5 \cdot 72$ \\
Second and third years & 208 & $14 \cdot 3$ \\
Infusion supplies (day) & - & $57 \cdot 2$ \\
RN (h) & 6 & - \\
RN/manager (h) & - & $5 \cdot 72$ \\
Unit clerk (h) & - & $7 \cdot 15$ \\
Scheduling clerk (h) & - & $5 \cdot 72$ \\
Ward aid (h) &
\end{tabular}

IVIG, intravenous immunoglobulin; RN, registered nurse; SCIG, subcutaneous immunoglobulin.

infusions and personnel. Rapid push SCIG infusion set supplies include butterfly needle, 12-cc syringe, alcohol swabs, gauze and paper tape $\left(6^{\prime \prime}\right)$. Supplies for IVIG infusion include IV tubing, D5W (5\% dextrose in water), Opsite, gauze, IV extension, max plus IV cap, over needle catheter (ONC), saline prefilled syringe and alcohol swabs. SCIG option requires a registered nurse manager (RN/Manager) for training patients $(6 \mathrm{~h}$ during the first year) and for yearly follow-up (6h per year). For IVIG, required personnel include a registered nurse (RN), a unit clerk, a scheduling clerk and a ward aid. The nurse for IVIG therapy is on duty for the full infusion period, whereas unit and scheduling clerks and ward aid are paid for 20,30 and $20 \mathrm{~min}$, respectively, for each visit regardless of duration of the infusion.

Unit costs for resources included in the model are shown in Table 2 and were obtained from St Paul's Hospital and the Adult SCIG home infusion program. All costs are in 2011 Canadian dollars. Cost of medication (immunoglobulin) was not included 
Table 2. Unit costs

\begin{tabular}{|c|c|c|}
\hline Parameter & $\begin{array}{l}\text { Unit cost } \\
\text { (Can\$) }\end{array}$ & Sources \\
\hline \multicolumn{3}{|l|}{ Supplies } \\
\hline $\begin{array}{l}\text { SCIG infusion set } \\
\text { Winged needle butterfly } \\
\text { (4 times/week) }\end{array}$ & $1 \cdot 15$ & $\begin{array}{l}\text { Ministry of Health, Product } \\
\text { Distribution Centre (2011) }\end{array}$ \\
\hline $\begin{array}{l}\text { IVIG infusion } \\
\quad \text { (total supplies) }\end{array}$ & $13 \cdot 54$ & $\begin{array}{l}\text { Ministry of Health, Product } \\
\text { Distribution Centre (2011) }\end{array}$ \\
\hline \multicolumn{3}{|l|}{ Personnel (h) } \\
\hline $\begin{array}{l}\mathrm{RN} / \text { manager } \\
\quad \text { (SCIG treatment) }\end{array}$ & $52 \cdot 5$ & $\begin{array}{l}\text { British Columbia Nurses' } \\
\text { Union (2011) }\end{array}$ \\
\hline RN (IVIG treatment) & $35 \cdot 00^{1}$ & $\begin{array}{l}\text { British Columbia Nurses' } \\
\text { Union (2011) }\end{array}$ \\
\hline Unit clerk & $19 \cdot 86$ & $\begin{array}{l}\text { Hospital Employees' Union } \\
\text { (2011) }\end{array}$ \\
\hline Scheduling clerk & $20 \cdot 77$ & $\begin{array}{l}\text { Hospital Employees' Union } \\
\text { (2011) }\end{array}$ \\
\hline Ward aid & $19 \cdot 86$ & $\begin{array}{l}\text { Hospital Employees' Union } \\
\text { (2011) }\end{array}$ \\
\hline
\end{tabular}

IVIG, intravenous immunoglobulin; RN, registered nurse; SCIG, subcutaneous immunoglobulin.

${ }^{1}$ Average wage.

in the model because it is the same for both IVIG and SCIG (Membe et al., 2008). As the SCIG winged needle butterfly infusion set does not require a pump, cost of the pump for IVIG was not included as a conservative approach to cost estimation. Parameters considered to have the greatest impact on model output were varied in sensitivity analyses including frequency of visits for IVIG treatment (13-17 visits) and duration of IVIG infusion (4-6h).

\section{Budget impact model}

A budget impact model (BIM) was developed to evaluate the annual budget impact of using SCIG instead of IVIG in adult PID patients. The epidemiological-based model included BC patients who received IVIG for PID from 1 April 2008 to 31 March 2009 according to BC Central Transfusion Registry (Provincial Health Services Authority, 2011). The analysis was performed from the healthcare system perspective and included the same direct medical costs as those considered in the cost-minimisation analysis over a period of 3 years. It was conservatively assumed that half $(50 \%)$ of patients on the registry switched from IVIG to SCIG based on data from a US survey, which reported that $75 \%$ of IVIG patients were willing to switch to SCIG percent (Ho et al., 2008), but that some of those patients were not eligible for SCIG. In the sensitivity analyses, base case results were compared with scenario A: rapid push SCIG versus least expensive option for IVIG (13 visits per year, 4-h infusion per visit); scenario B: rapid push SCIG versus most expensive IVIG option (17 visits per year, 6-h infusion per visit); and scenario C: $75 \%$ (instead of 50\%) switching to rapid push SCIG versus IVIG option used in the base case (14.3 visits per year, 4-h infusion per visit).

\section{RESULTS}

\section{Cost-minimisation analysis}

Under base case assumptions, SCIG treatment was estimated to cost $\$ 1978$ per patient over 3 years compared to $\$ 7714$ per patient for IVIG (Table 3). Using SCIG was less costly than IVIG treatment to the healthcare system by $\$ 5736$ per patient for the first 3 years of therapy. The main cost difference between the two options was personnel cost, which was greater for IVIG than SCIG: $\$ 2378$ for IVIG versus $\$ 630$ for the first year and $\$ 315$ for the second and third years of SCIG treatment. Yearly cost of disposable infusion supplies was slightly higher in SCIG (\$239) compared with IVIG (\$194). Results were sensitive to the number of visits during IVIG treatment, with SCIG less costly than IVIG over the first 3 years of therapy (savings of $\$ 5035$ vs IVIG-13 visits annually and of $\$ 7193$ vs IVIG-17 visits annually) (Table 4). Results were also sensitive to the duration of IVIG infusion during each visit with $\$ 5736$ lower cost over 3 years for SCIG versus IVIG (base case 4 -h infusion) and $\$ 8739$ lower cost when a 6-h infusion was employed.

\section{Budget impact model}

The population of PID patients on the BC registry for the fiscal year 2008-2009 was 456 (Provincial Health Services Authority, 2011). Under a conservative estimate of $50 \%$ of patients switching from IVIG of PID to SCIG, budget impact for the first 3 years after implementation of SCIG therapy was estimated to be $\$ 1.308$ million less for the base case scenario (Table 5). This represents a cost reduction of $37 \%$ of the total budget (all patients under IVIG therapy). This reduction in cost was \$1.148 million when SCIG was compared to the least expensive IVIG option (Scenario A: 13 visits and 4-h infusion) and $\$ 2.454$ million when it was compared to the most costly IVIG option (Scenario B: 17 visits and $6 \mathrm{~h}$ infusion). This represents 36 and $42 \%$ cost reduction, respectively, of the total budget. If the proportion of patients switching to SCIG was increased to $75 \%$ rather than $50 \%$ (Scenario C), \$1.962 million less would be required compared to the IVIG base case scenario ( $14 \cdot 3$ visits and 4 -h infusion), a cost reduction of $56 \%$ of total budget.

\section{DISCUSSION}

This study provides an economic evaluation of rapid push SCIG compared with IVIG treatment in adult PID patients from the perspective of the healthcare system. A cost-minimisation analysis demonstrated that rapid push SCIG is less costly than IVIG ( $\$ 1978$ compared to $\$ 7714$ per patient for the first 3 years of treatment), primarily because of less hospital personnel costs. This represents a cost difference between SCIG and IVIG treatments over 3 years of $\$ 5736$ or a $74 \%$ reduction. 
Table 3. Cost-minimisation model results (base case)

\begin{tabular}{|c|c|c|c|}
\hline Costs per patient & $\begin{array}{l}\text { SCIG } \\
(\text { Can\$ })\end{array}$ & $\begin{array}{l}\text { IVIG } \\
(\text { Can\$) }\end{array}$ & $\begin{array}{c}\text { Increment: } \\
\text { SCIG - IVIG } \\
(\text { Can\$) }\end{array}$ \\
\hline \multicolumn{4}{|l|}{ First year } \\
\hline Infusion supplies & 239 & 194 & 46 \\
\hline $\begin{array}{l}\mathrm{RN} \text { or RN/manager salary } \\
\text { Other personnel salary }\end{array}$ & 630 & 2002 & -1372 \\
\hline Unit clerk & - & 114 & \\
\hline Scheduling clerk & - & 149 & \\
\hline Ward aid & & 114 & \\
\hline Total personnel salary & 630 & 2378 & -1748 \\
\hline Total (first year) & 869 & 2571 & -1702 \\
\hline \multicolumn{4}{|l|}{ Second year } \\
\hline Infusion supplies & 239 & 194 & 46 \\
\hline $\begin{array}{l}\mathrm{RN} \text { or RN/manager salary } \\
\text { Other personnel salary }\end{array}$ & 315 & 2002 & -1687 \\
\hline Unit clerk & - & 114 & \\
\hline Scheduling clerk & - & 149 & \\
\hline Ward aid & - & 114 & \\
\hline Total personnel salary & 315 & 2378 & -2063 \\
\hline Total (second year) & 554 & 2571 & -2017 \\
\hline \multicolumn{4}{|l|}{ Third year } \\
\hline Infusion supplies & 239 & 194 & 46 \\
\hline $\begin{array}{l}\mathrm{RN} \text { or } \mathrm{RN} / \text { manager salary } \\
\text { Other personnel salary }\end{array}$ & 315 & 2002 & -1687 \\
\hline Unit clerk & - & 114 & \\
\hline Scheduling clerk & - & 149 & \\
\hline Ward aid & - & 114 & \\
\hline Total personnel salary & 315 & 2378 & -2063 \\
\hline Total (third year) & 554 & 2571 & -2017 \\
\hline Total cost per patient ( 3 years) & 1978 & 7714 & -5736 \\
\hline
\end{tabular}

IVIG, intravenous immunoglobulin; RN, registered nurse; SCIG, subcutaneous immunoglobulin.

Table 4 . Sensitivity analyses for the cost-minimisation model

\begin{tabular}{lcc}
\hline Parameter & Range & $\begin{array}{c}\text { Increment SCIG-IVIG } \\
\text { (Can\$ range) }\end{array}$ \\
\hline $\begin{array}{l}\text { Number of annual visits for } \\
\text { IVIG }\end{array}$ & $13-17$ & -5035 to -7193 \\
$\begin{array}{l}\text { Time of IVIG infusion per } \\
\text { visit (h) }\end{array}$ & $4-6$ & -5736 to -8739 \\
\hline
\end{tabular}

IVIG, intravenous immunoglobulin; SCIG, subcutaneous immunoglobulin.

In contrast with a previous Canadian economic study (Membe et al., 2008) the cost of immunoglobulin, which is the same for SCIG and IVIG in Canada, was not taken into account in the current analysis. Our study provides a comparison between the rapid push SCIG and IVIG options focused specifically on supplies and human resources, reflecting the context of the adult SCIG home infusion program based at St Paul's Hospital in Vancouver.
Results of this study are in agreement with one from the UK in which immunoglobulin costs for IVIG and SCIG were equivalent, which reported that SCIG was less costly than IVIG because hospital costs during IVIG therapy were greater than infusion pump and material required for SCIG treatment (Liu et al., 2005). In the UK study, annual costs were reduced by $88 \%$ for home-based SCIG compared with hospital-based IVIG with infusions every 3 weeks (Liu et al., 2005). In our study, 3-year costs of SCIG therapy compared with IVIG treatment with the same frequency (every 3 weeks) were reduced by $78 \%$. Other European studies comparing home-based SCIG and hospitalbased IVIG reported that the SCIG option was less costly principally because of less expensive immunoglobulin for SCIG (55-82\%) (Gardulf et al., 1995; Hogy et al., 2005) or to 31\% less immunoglobulin required to achieve therapeutic serum levels in SCIG compared with IVIG (Beaute et al., 2010).

Our study also evaluated the budgetary impact of switching $50 \%$ of the 456 adult PID patients in the BC Transfusion Registry from IVIG to SCIG treatment and found a reduction of $\$ 1.3$ million over 3 years for the healthcare system in BC. A panCanadian budget impact analysis, which considered the cost of immunoglobulin, reported annual savings for the healthcare systems of $\$ 6$ million ( $\$ 5.6$ million 2007Can\$ converted to 2011 Can\$) across Canada if $75 \%$ of 5460 adult patients switched from IVIG to SCIG treatment (Ho et al., 2008). In a German budget impact analysis, annual savings of $\$ 43$ million ( $\$ 28$ million $2003 €$ converted to 2011Can\$) from the perspective of the German statutory health insurance were reported for switching $60 \%$ of 2940 adult patients receiving IVIG treatment to SCIG but in this case immunoglobulin was much less costly for SCIG compared with IVIG (Hogy et al., 2005). The BIM presented here was sensitive to the proportion of patients switching from IVIG to SCIG suggesting the most economically efficient use of budget might be achieved by greater utilisation of SCIG therapy when clinically appropriate. Although difficult to quantify, freeing up of resources resulting from SCIG program could allow handling a greater number of interventions requiring IV infusions, such as chemotherapy.

From a patient perspective, the autonomy associated with SCIG administration translates into improved quality of life for PID patients. Studies using the Medical Outcomes Study 36Item Short Form Health Survey (SF-36) questionnaire to assess HRQoL and the Life Quality Index (LQI) to evaluate treatment satisfaction (TS) have reported that switching from hospitalbased IVIG to home-based SCIG improved both HRQoL and TS for adult PID patients significantly (Gardulf et al., 2004; Nicolay et al., 2006). In addition, SCIG delivered by rapid push has recently been shown to be preferred by adult PID patients who were given the choice (Misbah et al., 2009; Shapiro, 2010).

Typically, patients used SCIG delivered by the rapid push method 3.11 times a week for a 5-20 min infusion at one site each time achieving similar serum immunoglobulin levels and safety profile compared to patients who has chosen conventional pump infusion method (Shapiro, 2010). In this study, rapid push SCIG was delivered four times a week with similar infusion times. 
Table 5. Budget impact model results (base case and sensitivity analyses) (Can\$)

\begin{tabular}{|c|c|c|c|c|c|}
\hline Base case & Baseline & Year 1 & Year 2 & Year 3 & Total 3 years \\
\hline \% Switching (IVIG to SCIG) & - & 50 & 50 & 50 & - \\
\hline Number of patients IVIG & 456 & 228 & 228 & 228 & - \\
\hline Number of patients SCIG & 0 & 228 & 228 & 228 & - \\
\hline Total cost IVIG $(\$)$ & 1172525 & 586262 & 586262 & 586262 & 1758787 \\
\hline Total cost SCIG (\$) & 0 & 198178 & 126358 & 126358 & 450893 \\
\hline Incremental cost $(\$)$ & - & -388085 & -459905 & -459905 & -1307894 \\
\hline$\%$ Increment & - & -33 & -39 & -39 & -37 \\
\hline \multicolumn{6}{|c|}{ Sensitivity analysis A (IVIG 13 visits $-4 \mathrm{~h}$ ) } \\
\hline Total cost IVIG $(\$)$ & 1065931 & 532966 & 532966 & 532966 & 1598897 \\
\hline Total cost SCIG $(\$)$ & 0 & 198178 & 126358 & 126358 & 450893 \\
\hline Incremental cost (\$) & - & -334788 & -406608 & -406608 & -1148004 \\
\hline$\%$ Increment & - & -31 & -38 & -38 & -36 \\
\hline \multicolumn{6}{|c|}{ Sensitivity analysis B (IVIG 17 visits $-6 \mathrm{~h}$ ) } \\
\hline Total cost IVIG $(\$)$ & 1936550 & 968275 & 968275 & 968275 & 2904826 \\
\hline Total cost SCIG (\$) & 0 & 198178 & 126358 & 126358 & 450893 \\
\hline Incremental cost (\$) & - & -770098 & -841918 & -841918 & -2453933 \\
\hline$\%$ Increment & - & -40 & -43 & -43 & -42 \\
\hline \multicolumn{6}{|c|}{ Sensitivity analysis C (switching 75\%) } \\
\hline Number of patients IVIG & 456 & 114 & 114 & 114 & - \\
\hline Number of patients SCIG & 0 & 342 & 342 & 342 & - \\
\hline Total cost IVIG $(\$)$ & 1172525 & 293131 & 293131 & 293131 & 879393 \\
\hline Total cost SCIG $(\$)$ & 0 & 297266 & 189536 & 189536 & 676339 \\
\hline Incremental cost (4) & - & -582127 & -689857 & -689857 & -1961841 \\
\hline$\%$ Increment & - & -50 & -59 & -59 & -56 \\
\hline
\end{tabular}

IVIG, intravenous immunoglobulin; SCIG, subcutaneous immunoglobulin.

These results should be considered in light of study limitations. Because the most commonly used SCIG options in the SCIG home infusion program at St Paul's hospital does not required a pump, the cost of the SCIG pump was not included in the analyses. Considering a pump-based SCIG option would still result in savings to the healthcare system, estimated at \$1621 per patient and $\$ 369665$ in the budget impact analysis for the first 3 years of therapy compared to the IVIG treatment used in the base case even with the conservative approach of no cost for IVIG pump. This estimate assumed a weekly cost of infusion supplies of \$17.90 (Quadfurcated tubing safety Sub-Q infusion set) (RMS Medical Products, Chester, NY, US); that a SCIG pump lasts for 5 years and that two pumps are recommended for each patient (Hogy et al., 2005; Liu et al., 2005); that the type of infusion pump currently used at St Paul's Hospital costs $\$ 450.00$ (Freedom 60 Syringe driver), and adapting tubing for the pump costs $\$ 500.08$ per year [F900 Precision Infusion Tubing set (box of 50)] (RMS Medical Products, Chester, NY, US).

Another limitation relates to the fact that the models were designed to explore economic impact from the healthcare system perspective and therefore did not take into account costs borne by patients (parking and travel) and indirect costs such as productivity loss from patient and caregiver's time devoted to treatment. However, given the flexibility of home-based SCIG administration when considering the time, frequency and speed of infusion, patients' borne costs are minimal compared to hospital-based IVIG therapy. Finally, we did not consider the population of patients with secondary immune deficiencies which result from a variety of factors including infectious agents, drugs, metabolic diseases and environmental conditions (Chinen \& Shearer, 2010).

In conclusion, this study estimated that replacing IVIG with rapid push SCIG in $50 \%$ of adult PID patients resulted in 3 -year savings for the healthcare system of $\$ 5736$ per patient, representing reduced costs of $\$ 1.3$ million for the population of adult PID patients in British Columbia. Although focusing on the adult SCIG home infusion program based at St Paul's Hospital in Vancouver this study is in line with the current Canadian clinical practice and it can be expected that results would be generalisable to other Canadian settings. Hence, in addition to increased patient autonomy provided by SCIG compared with IVIG, shorter infusions better adapted to daily life of patients, and no need for a pump device, rapid push SCIG not only provides an improved option for patients but also results in significantly reduced costs from the healthcare system perspective in the Canadian context for immunoglobulin replacement therapy in adult PID patients. Further research incorporating societal costs and the population of secondary immune deficiencies patients could help achieve better insight into the economic consequences to be derived from use of the rapid push method for patients with immunoglobulin deficiency. 


\section{ACKNOWLEDGMENTS}

A. M. and R. S. contributed to the conception and design of the study and to the analysis and interpretation of data. A. M. collected the data. L. L. and M. G. contributed to the design of the analyses and interpretation of data and wrote the paper. R. S. and A. M. revised the manuscript critically for important intellectual content. All authors approved the final version of the manuscript submitted for publication. This study was made possible by a grant from CSL Behring Inc., Canada.

\section{CONFLICT OF INTEREST}

The authors have no competing interests.

\section{REFERENCES}

Beaute, J., Levy, P., Millet, V. et al. (2010) Economic evaluation of immunoglobulin replacement in patients with primary antibody deficiencies. Clinical and Experimental Immunology, 160, 240-245.

Berger, M. (2004) Subcutaneous immunoglobulin replacement in primary immunodeficiencies. Clinical Immunology, 112, 1-7.

Berger, M. (2008) Principles of and advances in immunoglobulin replacement therapy for primary immunodeficiency. Immunology and Allergy Clinics of North America, 28, 413-437.

British Columbia Nurses' Union. Wage grid. British Columbia Nurses' Union. 2011. URL http://www.bcnu.org/contracts_services/ provincial_contract/pdf/wage_grid.pdf (Accessed 14/12/11).

Chapel, H.M., Spickett, G.P., Ericson, D., Engl, W., Eibl, M.M. \& Bjorkander, J. (2000) The comparison of the efficacy and safety of intravenous versus subcutaneous immunoglobulin replacement therapy. Journal of Clinical Immunology, 20, 94-100.

Chinen, J. \& Shearer, W.T. (2010) Secondary immunodeficiencies, including HIV infection. The Journal of Allergy and Clinical Immunology, 125, S195-S203.

Gardulf, A., Andersen, V., Bjorkander, J. et al. (1995) Subcutaneous immunoglobulin replacement in patients with primary antibody deficiencies: safety and costs. Lancet, 345, 365-369.

Gardulf, A., Nicolay, U., Math, D. et al. (2004) Children and adults with primary antibody deficiencies gain quality of life by subcutaneous IgG self-infusions at home. The Journal of Allergy and Clinical Immunology, 114, 936-942.

Haddad, L., Perrinet, M., Parent, D., Leroy-Cotteau, A., Toguyeni, E., CondetteWojtasik, G. \& Hachulla, E. (2006) Economic evaluation of at home subcutaneous and intravenous immunoglobulin substitution. La Revue de Médecine Interne, 27, 924-926.

Ho, C., Membe, S., and Cimon, K. Subcutaneous versus intravenous immunoglobulin for primary immunodeficiencies: systematic review and economic evaluation. Technology report number 98. Canadian Agency for Drugs and Technologies in Health. 2008. URL http://cadth.ca/en/products/healthtechnology-assessment/publication/785 (Accessed 27/10/11).

Hogy, B., Keinecke, H.O. \& Borte, M. (2005) Pharmacoeconomic evaluation of immunoglobulin treatment in patients with antibody deficiencies from the perspective of the German statutory health insurance. The European Journal of Health Economics, 6, 24-29.

Hospital Employees' Union. Wage scedules - Schedule B - Rate levels. 2010-2012 Collective agreement. General wage schedule. Hospital Employees' Union. 2011. URL http://www.heu.org/sites/default/files/ uploads/collective_agreement/2011/03/16/ Facilities\%202010\%20to\%202012\%20wage \%20rates.pdf (Accessed 14/12/11).

Kittner, J.M., Grimbacher, B., Wulff, W., Jager, B. \& Schmidt, R.E. (2006) Patients' attitude to subcutaneous immunoglobulin substitution as home therapy. Journal of Clinical Immunology, 26, 400-405.

Lemieux, R., Bazin, R. \& Neron, S. (2005) Therapeutic intravenous immunoglobulins. Molecular Immunology, 42, 839-848.

Liu, Z., Albon, E., and Hyde, C. The effectiveness and cost effectiveness of immunoglobulin replacement therapy for primary immunodeficiency and chronic lymphocytic leukaemia: a systematic review and economic evaluation. The University of Birmingham. 2005. URL www.rep. bham.ac.uk/2005/IgRT.pdf (Accessed 19/10/11).
Membe, S.K., Ho, C., Cimon, K., Morrison, A., Kanani, A. \& Roifman, C.M. (2008) Economic assessment of different modalities of immunoglobulin replacement therapy. Immunology and Allergy Clinics of North America, 28, 861-874.

Misbah, S., Sturzenegger, M.H., Borte, M., Shapiro, R.S., Wasserman, R.L., Berger, M. \& Ochs, H.D. (2009) Subcutaneous immunoglobulin: opportunities and outlook. Clinical and Experimental Immunology, 158 (Suppl. 1), 51-59.

Nicolay, U., Kiessling, P., Berger, M. et al. (2006) Health-related quality of life and treatment satisfaction in North American patients with primary immunedeficiency diseases receiving subcutaneous IgG selfinfusions at home. Journal of Clinical Immunology, 26, 65-72.

Product Distribution Centre. 2011. URL http://www.pdc.gov.bc.ca/Account/LogOn (Accessed 13/12/11).

Provincial Health Services Authority. Provincial blood coordinating office. Provincial Health Services Authority. 2011. URL http:// www.phsa.ca/AgenciesAndServices/ Services/PBCO.htm (Accessed 11/11/11).

Shapiro, R. (2010) Subcutaneous immunoglobulin therapy by rapid push is preferred to infusion by pump: a retrospective analysis. Journal of Clinical Immunology, 30, 301-307.

Shehata, N., Palda, V., Bowen, T. et al. (2010) The use of immunoglobulin therapy for patients with primary immune deficiency: an evidence-based practice guideline. Transfusion Medicine Reviews, 24 (Suppl. 1), S28-S50.

Sorensen, R.U. \& Moore, C. (2000) Antibody deficiency syndromes. Pediatric Clinics of North America, 47, 1225-1252.

Turvey, S.E., Bonilla, F.A. \& Junker, A.K. (2009) Primary immunodeficiency diseases: a practical guide for clinicians. Postgraduate Medical Journal, 85, 660-666. 